Running head: tDCS and affective switching

\title{
Prefrontal transcranial direct current stimulation facilitates affective flexibility
}

\author{
Tatiana Aboulafia-Brakha ${ }^{1,2,3}$; Aurelie L. Manuel ${ }^{2}$ and Radek Ptak ${ }^{1,2,3}$
}

${ }^{1}$ Division of Neurorehabilitation, Department of Clinical Neurosciences, Geneva University Hospitals, Switzerland

${ }^{2}$ Laboratory of Cognitive Neurorehabilitation, Department of Clinical Neurosciences, Medical School, University of Geneva, Geneva, Switzerland

3Faculty of Psychology and Educational Sciences, University of Geneva, Geneva, Switzerland

Word count: Abstract: 200, Manuscript: 4’202

Corresponding author: Tatiana Aboulafia Brakha, Division of Neurorehabilitation,

Department of Clinical Neurosciences, Geneva University Hospitals, Av. De Beau-Séjour 26, CH-1211 Geneva 14, Switzerland. Tel: $+41-22-372860$.

E-mail: Tatiana.Aboulafia@hcuge.ch 


\begin{abstract}
Performance on paradigms involving switching between emotional and non-emotional task-sets (affective flexibility) predicts emotion regulation abilities and is impaired in patients with different emotional disorders. A better understanding of how neurostimulation techniques such as transcranial direct current stimulation (tDCS) influence affective switching may provide support for the improvement of rehabilitation programs. In the current study healthy volunteers received anodal tDCS over the right dorsolateral prefrontal cortex (DLPFC), the left DLPFC or sham stimulation while performing an affective-switching task. Participants had to repeat or switch between facial judgments of emotional expressions (emotional task-set) or gender (non-emotional task-set). Right tDCS resulted in faster responses in the gender task only when it followed a judgment of emotion. These effects were not observed following left tDCS. Further, switching away from emotion was easier for the right compared to left tDCS group (reduced switch costs for gender), while switching away from gender toward emotion was easier for the left compared to the right group (reduced switch-costs for emotion). In sum, tDCS over the DLPFC may modulate affective flexibility and right stimulation may be particularly helpful to facilitate disengagement from emotional task-sets. The usefulness of tDCS-trained affective switching may be further investigated on larger therapeutic protocols targeting emotional disorders.
\end{abstract}

Keywords: Affective flexibility, emotion regulation, neurostimulation, switching, transcranial direct current stimulation. 


\section{Prefrontal transcranial direct current stimulation facilitates affective flexibility}

\section{INTRODUCTION}

Rapid identification of emotional facial expressions is an essential, socially adaptive skill. It allows adequate response to an interlocutor during interpersonal exchanges (Kret \& Ploeger, 2015) for review. However, being able to disengage attention from emotional features and orienting toward non-emotional information is also required in order to cope with socially distressing situations and emotional events in general (de Vries \& Geurts, 2012; Genet \& Siemer, 2011; Johnson, 2009). Attending to and disengaging from emotional material in a flexible manner is termed affective flexibility or affective switching (Malooly, Genet, \& Siemer, 2013). This ability is associated with the use of adaptive emotion regulation strategies, such as reappraisal - the ability to cognitively re-interpret the situation and thus to

modify emotional experience (J. J. Gross, 2002; Malooly et al., 2013). Affective switching ability and the frequent use of reappraisal are both predictors of lower levels of anxiety and depression (De Lissnyder et al., 2012; Johnson, 2009).

Experimental paradigms focusing on affective flexibility are good predictors of the implementation of these skills in everyday life (De Lissnyder et al., 2012; Genet \& Siemer, 2011). For instance, Genet and Siemer (2011) examined the relationship between selfreported flexible adaptation to emotional events in daily life and performance on a task involving switching between categorizing affective words according to their valence or grammatical class. They found that emotional flexibility in everyday life predicted better switching abilities, as shown by lower task switch-costs. Switch-costs reflect the difference between reaction times on trials when a task is repeated and trials requiring a switch (Monsell, 2000). Further support for the clinical importance of affective switching paradigms is 
provided by studies showing that this ability is diminished in clinical samples characterized by difficulties in social adaptation. For example, in contrast to controls, children with autism spectrum disorders showed slower reaction times when switching from emotional to nonemotional facial features (de Vries \& Geurts, 2012). This was not observed for switches occurring in the reverse direction. In a similar paradigm, patients with bipolar disorder showed greater switch-costs (higher reaction times on switch compared to non-switch trials) than controls when gender judgment of faces followed the judgment of their emotional valence (Gul \& Khan, 2014). Furthermore, affective switching not only predicts poorer flexibility in managing emotions in everyday life, but is also associated with less adaptive responses to stressful events (De Lissnyder et al., 2012).

Standard therapeutic programs targeting emotion regulation or social skills may improve affective switching in everyday life and lead to better social adaptation (Goldin et al., 2014; Ponizovsky et al., 2013; Wyman et al., 2010). Moreover, as demonstrated in the treatment of depression or rehabilitation following brain injury, effects of these standard programs may be enhanced when combined with noninvasive neuromodulation techniques, such as transcranial direct current stimulation (tDCS) (Dhaliwal, Meek, \& Modirrousta, 2015; Meron, Hedger, Garner, \& Baldwin, 2015; Shiozawa et al., 2014). At a neural level, tDCS modifies excitability of neurons under the targeted cortical area, increasing it with anodal (excitatory) stimulation and decreasing it with cathodal (inhibitory) stimulation (Bikson, Name, \& Rahman, 2013; Nitsche \& Paulus, 2000; Tremblay et al., 2014). Boggio and colleagues (2015) argue that tDCS is a promising tool in the rehabilitation of social deficits. Ideally, investigation protocols should start by examining short-term effects of neurostimulaton, as observed in a single stimulation session, and move toward the investigation of more long lasting effects (repetitive stimulation or inclusion of a stimulation session in larger therapeutic protocols) (Boggio et al., 2015). 
The choice of the region to be stimulated is usually based on functional neuroimaging and electrophysiological data using similar paradigms or on reported effects of previous stimulation. For instance, studies using functional imaging report bilateral activation of the dorsolateral prefrontal cortex (DLPFC) during task-switching for emotional and nonemotional material (Loose, Kaufmann, Tucha, Auer, \& Lange, 2006; Piguet et al., 2013). However, there appears to be a hemispheric difference, with the left DLPFC being preferentially activated by non-emotional tasks (Ravizza \& Carter, 2008; Wendelken, Munakata, Baym, Souza, \& Bunge, 2012; Yoshida, Funakoshi, \& Ishii, 2010) and the right DLPFC for tasks implicating affective switching (Krug \& Carter, 2012; Reeck \& Egner, 2014). Consistent with neuroimaging studies, excitatory anodal tDCS applied over the left DLPFC improved switching in a paradigm using non-emotional verbal material (naming letters or digits according to specific cues) (Leite, Carvalho, Fregni, Boggio, \& Goncalves, 2013). Stimulating the same region, Vanderhasselt et al. (2013) found improved reaction times in a task requiring to evoke the opposite emotion to the one depicted by a face (e.g., 'sadness' when the image showed a happy face). This study did not examine switches between emotional and non-emotional tasks and did not include a control condition with right DLPFC stimulation. Performance on affective switching paradigms is known to be related to emotion regulation abilities, and these have been reported to improve following tDCS over the right DLPFC (De Lissnyder et al., 2012; Feeser, Prehn, Kazzer, Mungee, \& Bajbouj, 2014; Genet \& Siemer, 2011; Riva, Romero Lauro, DeWall, Chester, \& Bushman, 2015). However, none of these previous studies compared the influence of tDCS over the left and the right DLPFC on competition between emotional and non-emotional task-sets.

Here, we examined the effects of anodal-excitatory tDCS over the left and right DLPFC on 1) task repetition involving switching within emotional and non-emotional tasksets (e.g., from joy to anger and vice versa, and from male to female and vice versa), 2) 
switching between emotional and non-emotional tasks sets, 3 ) task switch-costs (reaction time differences between task-switching and task-repetition). Given the strong implication of the DLPFC in task switching we hypothesized that anodal stimulation of this region would facilitate switching processes.

\section{MATERIAL AND METHODS}

\subsection{Participants}

Forty-five healthy volunteers (21 males, 42 right-handed), aged between 19 and 52 years $($ Mean $=27.16, \mathrm{SD}=6.7)$ participated for payment to this study. They reported no previous psychiatric or neurological disease and had normal or corrected-to-normal visual acuity. Once they agreed to participate, they were successively allocated to one of the three stimulation groups: right anodal tDCS, left anodal tDCS, or sham. Participants were blind to condition, but not the administrator. The three groups did not differ significantly regarding gender distribution $\left(X^{2}(2)=4.8, n s\right)$ or age $(F(2,42)=1.8, n s$; Right: Mean $=24.5, S D=3.6$; Left: Mean $=28.3, S D=6.3$; Sham Mean $=28.6 \mathrm{SD}=8.7$ ). As shown in Table 1, groups did not differ regarding self-reported depressive symptoms on the Beck Depression Inventory-2-BDI (Beck, Ward, Mendelson, Mock, \& Erbaugh, 1961) $(F(2,41)=0.36, n s)$, proneness to anger manifestations on the The Spielberger trait-anger inventory-STAXI-T 2 (Spielberger, Reheiser, \& Sydeman, 1995) $(F(2,42)=1.24, n s)$ or use of emotion regulation strategies on the Emotion Regulation Questionnaire-ERQ (J. Gross \& Oliver, 2002) (Reappraisal: F $(2,36)$ $=0.49, \mathrm{~ns} ;$ Suppression: $F(2,36)=1.25, n s)$.

\subsection{Material and procedures}

The study was approved by the local ethics committee. In a single experimental session, participants signed the informed consent, filled out a safety-screening health 
questionnaire (in order to verify suitability to tDCS) and completed an affective switching task under one of the three stimulation conditions. They then completed a demographic questionnaire and self-report psychological scales.

\subsubsection{Affective switch Task}

Stimuli and task requirement: The stimulus set was composed of 12 different faces (6 males, 6 females; 6 happy and 6 angry) from the Karolinska Directed Emotional Faces database (Lundqvist et al., 1998). Four different stimuli were created with each face (dimensions: $9 \times 14 \mathrm{~cm}$ ) by placing four different verbal tags ('male', 'female', 'Joy' or 'anger') above the face (see Figure 1), resulting in 48 different stimuli. The tag indicated whether participants should make a judgment concerning the expressed emotion (happy, angry) or the gender (male, female) of the depicted face. When the face shown on screen was congruent with the task label, either regarding emotion or gender, a go-response was required. If the face was incongruent with the tag no response was expected. In order to minimize the demands on working memory the tag (which served as task cue) was presented simultaneously with the face on white background on a 19" computer screen. Importantly, the task was designed in a Go/no-go format in order to avoid potential confounds of affective switches with manual or digital switches (which would be the case if go-responses were required for trials in which stimuli were incongruent with labels).

Procedure: Stimuli were presented for $2000 \mathrm{~ms}$ and were replaced by a fixation cross between trials for $500 \mathrm{~ms}$. Participants were placed at approximately $70 \mathrm{~cm}$ distance of the screen and were instructed to press the space bar (go-response) with their dominant hand. Given the requirement to include a similar number of repetition and switch trials stimuli were presented in pseudorandomized sequence. Repetition trials occurred when a judgment for the same task had to be performed twice in succession (i.e., two gender judgment or two emotion judgments in sequence). Switch trials were characterized by a change of task (i.e., an emotion 
judgment following a gender judgment or vice versa). Participants performed two blocks of 192 trials, each containing 112 no-go trials and 80 go-trials. Of these go-trials, 40 were repetition trials and 40 switch trials; with a balanced number of emotion and gender trials in each category, yielding 20 trials per subcategory in each block. The duration of one block was approximately 10 minutes, and the two blocks were separated by a rest of 2-3 minutes. Prior to the start of block one all participants completed a 48-trial practice block with the same stimuli. The design was chosen to ensure that the whole experiment was finished within 25 min online tDCS stimulation.

\subsection{2 tDCS}

Two sponged electrodes ( 7 X $5 \mathrm{~cm}$ ) soaked in an isotonic solution (NaCL $0.9 \%$ ) were connected to a battery driven DC-stimulator (Neuroconn, Illmenau, Germany) that delivered the intended current. In the right anodal group, the anodal electrode was placed over the right DLPFC (electrode position F4 according to the 10-20 EEG system) and the contralateral cathodal electrode over the left supraorbital area (position FP1). In the left anodal group the anodal electrode was placed over the left DLPFC (position F3) and the cathodal electrode over the right supraorbital area (position FP2).

In these two groups, a current flow of $1.5 \mathrm{~mA}$ was applied during 25 minutes. Of note, intensity, duration and the size of electrodes met international safety standards (Bikson, Datta, \& Elwassif, 2009). In a third group (SHAM), participants had the same electrode montages and voltage as the two stimulation groups (in alternation), but stimulation was only delivered for 30 seconds and then turned off. In all groups current was delivered with eight seconds of ramp-up and ramp-down period. Participants of the active tDCS groups started the task five minutes after stimulation onset and completed it before its end.

\subsection{Statistical Analysis}


Mixed ANOVAs with group (anodal right, anodal left, sham) and task (emotion, gender) as factors were performed on mean reaction times (RTs) from switch trials and repetition trials. Switch costs were computed by subtracting RTs on repetition trials from RTs on switch trials and analyzed these with 3 (group) x 2 (task) mixed ANOVAs. Since the whole experiment lasted at least 20 minutes we also repeated the analyses separately for each block due to the commonly found learning effects in task-switching paradigms. To do so, we performed the primary analyses on results of the two blocks (identical sequence and stimuli) separately. Significance levels were set to $p<0.05$. Significant interactions were followed-up with independent $t$-tests for between-group comparisons and paired $t$-tests for within-group contrasts. In these cases we used Bonferroni-corrected alpha-levels $(p<0.005)$.

\section{RESULTS}

\subsection{Effects of tDCS on emotional and non-emotional task-repetition trials}

The 3 (Group: anodal right, anodal left, sham) x 2 (Task: emotion, gender) ANOVA on mean RTs showed no main effect of group $(F(2,42)=0.86, p=0.43)$, but a main effect of task $\left(F(1,42)=30.40, p<0.00001, \eta \rho^{2}=0.42\right.$; Figure 2$)$. The interaction between group and task was not significant $(F(1,42)=0.34, p=0.71)$. The analysis performed on the two blocks (Table 1) separately revealed the same pattern observed in the whole experiment, with a significant main effect of task (Block 1: $F(1,42)=20.27, p<0.00001$, Block 2: $F(1,42)=$ 13.55, $p<0.001)$, but no significant main effect of group (Block 1: $F(2,42)=1.03, p=0.37$; Block 2: $\mathrm{F}(2,42)=0.69, p=0.50)$ and no interaction between group and task (Block 1: $F(2$, $42)=0.56, p=0.57) ;$ Block 2: $F(2,42)=1.23, p=0.30)$.

In sum, tDCS did not yield differential effects on task-repetition trials.

\subsection{Effects of tDCS on switching between emotional and non-emotional tasks}


The 3 (Group: anodal right, anodal left, sham) x 2 (Task: emotion, gender) ANOVA revealed no significant main effect of group $(F(2,42)=0.64, p=0.53)$, but a main effect of task $\left(F(1,42)=12.74, p=0.001, \eta \rho^{2}=0.23\right)$ and a significant interaction between group and task $\left(F(2,42)=3.18, \mathrm{p}=0.05, \eta \rho^{2}=0.13\right)$ suggesting that tasks were differentially affected by stimulation (Figure 2). Post-hoc comparisons regarding between-group effects of tDCS for emotion trials (following gender) and gender trials (following emotion) were not significant $(0.16<p<0.85)$. Post-hoc within-group contrasts showed significantly shorter RTs for gender trials (following emotion) compared to emotion trials (following gender) in the right anodal group $(t(14)=6.17, p<0.0001)$, a trend (considering Bonferroni-corrected alphalevel) in the same direction in the sham group $(t(14)=2.86, p=0.01)$ and no significant differences between tasks in the left anodal group $(t(14)=0.20, p=0.84)$.

The 3 (Group: anodal right, anodal left, sham) x 2 (Task: emotion, gender) ANOVA on RTs during block 1 (Table 1 ) showed no significant effects of group $(\mathrm{F}(2,42)=0.66, p=$ $0.52)$, but a significant main effect of task $(F(1,42)=26.64, p<0.00001)$ and a significant interaction between group and task $(F(2,42)=3.22, p=0.05)$. The analysis performed on block 2 (Table 1) yielded no significant results (Group: $F(2,42)=0.59, p=0.56)$, Task: $F(1$, $42)=0.85, p=0.36)$; interaction between group and task: $F(2,42)=1.41, p=0.25)$.

To summarize, the group receiving right anodal $t D C S$ showed shorter RTs for gender judgments when these followed an emotion judgment than for emotion judgment when these followed a gender judgment. No differential effects were observed in the left anodal $t$ DCS. Further, effects observed in the whole experiment seem to be driven by results in the first block. 


\subsection{Effects of tDCS on Switch costs}

Switch costs were calculated by subtracting mean RTs on task-repetition trials from RTs on task-switch trials for emotion and gender tasks separately (Figure 3). Thus, a positive switch cost indicates that participants were slower to switch tasks relative to repeating the same task, while a negative switch cost indicates that switching tasks was faster than repeating tasks. The $3 \times 2$ ANOVA on total RTs showed no significant effect of group $(F(2$, $42)=0.36, p=0.69)$ or task $(F(1,42)=0.85, p=0.36)$, nor a significant interaction $(F(2$, $42)=2.00, p=0.15)$. However, the $3 \times 2$ ANOVA performed on RTs of block 1 showed a significant interaction between group and task $(F(2,42)=3.12, p=0.05)$, but no significant main effect of group $(F(2,42)=0.53, p=0.59)$ or task $(F(1,42)=1.29, p=0.26)$. Post-hoc independent $\mathrm{t}$-tests revealed that the interaction was driven by a trend (considering Bonferroni-corrected alpha-level) to differential effects of right and left tDCS on emotion and gender switch costs. For instance, switch-costs for emotion trials were smaller in the left compared to the right tDCS group $(t(28)=2.08, p=0.05)$, suggesting that it was easier to switch from gender, while switch-costs for gender trials were smaller in the right compared to the left stimulation group $(t(28)=-2.03, p=0.05)$, suggesting that it was easier to switch away from emotion. In addition, there was a trend toward a within-group difference in the right stimulation group, with lower switch costs for gender trials compared to emotion trials $(t$ $(14)=2.56, p=0.02)$. The remaining within $(p>0.13)$ and between (all $p>0.29)$ group contrasts did not reach significance. In block 2 the $3 \times 2$ mixed measure ANOVA showed no significant effect of group $(F(2,42)=0.13, p=0.88)$, nor a significant interaction between group and task $(F(2,42)=0.57, p=0.57)$, but a significant main effect of task $(F(1,42)=$ $3.99, p=0.05)$. 
Thus, the main finding of these analyses was that switch-costs for gender were reduced in the right tDCS group compared to the left tDCS group, while for emotion these group differences were reversed.

\section{DISCUSSION}

We examined the influence of anodal tDCS targeting the DLPFC on switching between emotional and non-emotional tasks-sets, an ability correlated to the use of adaptive emotion regulation strategies (J. J. Gross, 2002; Malooly et al., 2013). Effects of both right and left anodal tDCS on switch costs for emotional and non-emotional task-sets suggest that tDCS over the DLPFC may modulate affective flexibility. While left tDCS seems to facilitate switching toward emotional tasks-sets, right anodal tDCS facilitates switching in the opposite direction, by enhancing disengagement from emotional task-sets. More specifically, right anodal tDCS results in faster judgment of non-emotional features (gender) when the task required switching from a judgment of emotional expression. On the other hand, this group showed increased switch-costs for emotion. We will argue below that the latter finding suggests increased inhibition of emotional task-sets (Monsell, Yeung, \& Azuma, 2000).

The affective-switching task we used in the current study included task-repetition trials (gender to gender and emotion to emotion) and task-switch trials (from emotion to gender and vice-versa). Similar paradigms have previously been used in behavioral or fMRI studies (de Vries \& Geurts, 2012; Piguet et al., 2013; Reeck \& Egner, 2014), but none of them examined performance under a neurostimulation technique. Interestingly, while we observed no tDCS effects on gender or emotion repetition trials, the right anodal TDCS group showed faster judgment of gender features only when these followed an emotion judgment. Further, right tDCS seems to emphasize effects of naturalistic performance (without stimulation) of the sham group, which showed a tendency to a faster switch toward non-emotional task-sets. As no effects of tDCS were observed on repetition trials, facilitation effects observed for 
gender judgment in the right anodal tDCS are specifically related to the transition from emotion task-sets rather than to gender judgment per se.

Studies on task-switching indicate that an inhibition mechanism is implicated in switch processes in the sense that the previous task-set must be inhibited in order to make the subsequent task accessible (Koch, Gade, Schuch, \& Philipp, 2010; Monsell, Sumner, \& Waters, 2003; Monsell et al., 2000). As a consequence, the stronger a task-set is inhibited, the easier it becomes to switch to a subsequent task (Monsell et al., 2000). Importantly, returning to a previously inhibited task-set becomes harder and increased reaction times are commonly observed (Monsell et al., 2000; Wylie and Allport, 2000). In the current study, right anodal tDCS may have favored inhibition of emotional task-set in order to facilitate switch to nonemotional task-set. In agreement with this finding, the analyses of switch-costs showed increased switch-costs for emotion task-sets (i.e., it was harder to switch to emotion) and reduced switch-costs for gender (it was easier to switch from emotion).

Interestingly, tDCS applied to left and right DLPFC engendered opposite effects on switch costs. The left anodal group showed reduced switch-costs for emotion compared to the right tDCS group, suggesting that it was easier to switch from a non-emotional task-set to an emotional task-set. Conversely, switch-costs for gender were smaller in the right compared to left tDCS group. This latter finding implies that it is easier to disengage attention from an emotional task-set toward a non-emotional task-set following right anodal tDCS. This facilitation effect observed with our healthy participants goes exactly in the opposite direction to naturalistic impaired performance of patients with bipolar disorder or autism spectrum disorders in paradigms similar to ours (de Vries \& Geurts, 2012; Gul \& Khan, 2014). For instance, these two groups of patients show greater switch costs for non-emotional task-sets (gender) as a consequence of a difficulty to disengage attention from emotional task-sets. This imbalance was not observed in controls. In addition, performance of patients with bipolar 
disorders on the affective switching paradigm was predicted by the ability to use emotion regulation strategies in everyday life (Gul \& Khan, 2014). Given the tight relation between affective switching and emotion regulation our findings are also consistent with studies reporting that right anodal tDCS facilitates emotion regulation processes (Feeser et al., 2014; Pripfl \& Lamm, 2015; Riva et al., 2015).

One question is whether the facilitation of emotional disengagement we observed following right anodal tDCS could be explained by better global emotional processing, a function that strongly depends on a right hemispheric network (Gainotti, 2012; Shobe, 2014). However, no effects of $t$ DCS on emotion judgment per se were found on repetition or switchtrials. This finding appears to contradict a recent tDCS study showing that right anodal stimulation decreases reaction time (and increases accuracy) for emotion recognition (Willis, Murphy, Ridley, \& Vercammen, 2015). However, if the paradigm focuses on a single task-set (e.g., only emotion task) behavioral response patterns and underlying neural mechanisms are different than if it implicates switching between task-sets, even if stimuli are identical (Reeck $\&$ Egner, 2014). This may explain the absence of right $t$ DCS effects on emotion judgment in our study. An additional difference to the study of Willis and collaborators (2015) was that these authors applied tDCS over the orbito-frontal cortex, which may have had stronger effects on emotion recognition.

Regarding neural mechanisms implicated in affective task-switching, our findings corroborate previous neuroimaging data reporting activation of the right DLFPC in affective switching tasks (Krug \& Carter, 2012; Reeck \& Egner, 2014). Our results are also consistent with a lesion study reporting impaired switching in patients with right frontal damage which was due specifically to poor task-set inhibition (Aron, Monsell, Sahakian, \& Robbins, 2004). Patients with left frontal damage also showed impaired switching, but this was attributed to impaired task control rather than inhibition. Given that in this study the task involved non- 
emotional material these findings raise the question whether increased excitability of the right DLPFC specifically contributes to better affective switching or to facilitation of switching in general. However, studies examining the effects of prefrontal tDCS on non-emotional switching only found stimulation effects when tDCS was applied over the left, but not the right DLPFC (Leite et al., 2013; Vanderhasselt et al., 2013). Together with our study these findings suggest distinct and possibly independent neural mechanisms implicated in affective and non-affective flexibility.

While the main results of our study were maintained across the whole stimulation period (block 1 and block 2 conjoined), some findings were driven by performance observed during the first block. This may suggest that effects of $t$ DCS are strongest during the first minutes of stimulation, or may reflect effects of practice (Chan, Shum, Toulopoulou, \& Chen, 2008). Practicing an executive task not only leads to performance improvement, but also to a shift in brain mechanisms allowing to execute the same task with greater automaticity (Collette, Hogge, Salmon, \& Van der Linden, 2006; Collette et al., 2005). Thus, the decrease of effects in the second block might reflect lesser importance of the right DLPFC for emotional switching with practice. Further, we cannot rule-out a "state-dependency effect" (Filmer, Dux, \& Mattingley, 2014). As tDCS effects depend on the activity of the stimulated cortical area, it is possible that when a particular cognitive effort related to switching is no longer needed (due to practice) tDCS induces weaker effects. In addition, the absence of effects across the whole experiment could also be due to our limited sample-size in this between-subject design. Finally, we cannot exclude that group differences were present independently of stimulation. However between-group differences were not observed on repetition trials and only emerged on switch trials in interaction with task, which makes it more likely that performance was affected by stimulation. 


\section{CONCLUSION AND PERSPECTIVES}

Processing emotional features is important in everyday life, while inhibiting them allows us to focus on other important information. By applying anodal tDCS over the right DLPFC we observed that switching processes for emotional material can be modulated. While left anodal tDCS over the DLPFC may potentially facilitate deployment of attention toward emotional task-sets, right anodal tDCS may facilitate disengagement from it. These findings are particularly important with regard to the treatment of emotion regulation in clinical samples. Future studies might examine the effects of tDCS in patients with impaired emotion processing or regulation as a part of treatment protocols, in isolation or accompanying traditional psychotherapeutic interventions.

Acknowledgement: Study supported by the Swiss National Science Foundation (grant PMCDP1_151305 to TAB). We declare no conflict of interest.

\section{References}

Aron, A. R., Monsell, S., Sahakian, B. J., \& Robbins, T. W. (2004). A componential analysis of task-switching deficits associated with lesions of left and right frontal cortex. Brain, 127(Pt 7), 1561-1573. doi: 10.1093/brain/awh169

Beck, A. T., Ward, C. H., Mendelson, M., Mock, J., \& Erbaugh, J. (1961). An inventory for measuring depression. Arch Gen Psychiatry, 4, 561-571.

Bikson, M., Datta, A., \& Elwassif, M. (2009). Establishing safety limits for transcranial direct current stimulation. Clin Neurophysiol, 120(6), 1033-1034. doi: 10.1016/j.clinph.2009.03.018

Bikson, M., Name, A., \& Rahman, A. (2013). Origins of specificity during tDCS: anatomical, activity-selective, and input-bias mechanisms. Front Hum Neurosci, 7, 688. doi: 10.3389/fnhum.2013.00688

Boggio, P. S., Asthana, M. K., Costa, T. L., Valasek, C. A., \& Osorio, A. A. (2015). Promoting social plasticity in developmental disorders with non-invasive brain stimulation techniques. Front Neurosci, 9, 294. doi: 10.3389/fnins.2015.00294

Chan, R. C., Shum, D., Toulopoulou, T., \& Chen, E. Y. (2008). Assessment of executive functions: review of instruments and identification of critical issues. Arch Clin Neuropsychol, 23(2), 201-216. doi: 10.1016/j.acn.2007.08.010 
Collette, F., Hogge, M., Salmon, E., \& Van der Linden, M. (2006). Exploration of the neural substrates of executive functioning by functional neuroimaging. Neuroscience, 139(1), 209-221. doi: 10.1016/j.neuroscience.2005.05.035

Collette, F., Van der Linden, M., Laureys, S., Delfiore, G., Degueldre, C., Luxen, A., \& Salmon, E. (2005). Exploring the unity and diversity of the neural substrates of executive functioning. Hum Brain Mapp, 25(4), 409-423. doi: 10.1002/hbm.20118

De Lissnyder, E., Koster, E. H., Goubert, L., Onraedt, T., Vanderhasselt, M. A., \& De Raedt, R. (2012). Cognitive control moderates the association between stress and rumination. $J$ Behav Ther Exp Psychiatry, 43(1), 519-525. doi: 10.1016/j.jbtep.2011.07.004

de Vries, M., \& Geurts, H. M. (2012). Cognitive flexibility in ASD; task switching with emotional faces. J Autism Dev Disord, 42(12), 2558-2568. doi: 10.1007/s10803-012$1512-1$

Dhaliwal, S. K., Meek, B. P., \& Modirrousta, M. M. (2015). Non-Invasive Brain Stimulation for the Treatment of Symptoms Following Traumatic Brain Injury. Front Psychiatry, 6, 119. doi: 10.3389/fpsyt.2015.00119

Feeser, M., Prehn, K., Kazzer, P., Mungee, A., \& Bajbouj, M. (2014). Transcranial direct current stimulation enhances cognitive control during emotion regulation. Brain Stimul, 7(1), 105-112. doi: 10.1016/j.brs.2013.08.006

Filmer, H. L., Dux, P. E., \& Mattingley, J. B. (2014). Applications of transcranial direct current stimulation for understanding brain function. Trends Neurosci, 37(12), 742753. doi: 10.1016/j.tins.2014.08.003

Gainotti, G. (2012). Unconscious processing of emotions and the right hemisphere. Neuropsychologia, 50(2), 205-218. doi: 10.1016/j.neuropsychologia.2011.12.005

Genet, J. J., \& Siemer, M. (2011). Flexible control in processing affective and non-affective material predicts individual differences in trait resilience. Cogn Emot, 25(2), 380-388. doi: 10.1080/02699931.2010.491647

Goldin, P. R., Lee, I., Ziv, M., Jazaieri, H., Heimberg, R. G., \& Gross, J. J. (2014). Trajectories of change in emotion regulation and social anxiety during cognitivebehavioral therapy for social anxiety disorder. Behav Res Ther, 56, 7-15. doi: 10.1016/j.brat.2014.02.005

Gross, J, \& Oliver, J. (2002). Wise emotion regulation. In L. F. Barrett \& Salovey; P. (Eds.), The wisdome of feeling : Psychological processes in emotional

intelligence (pp. 297-319). New York: Guilford.

Gross, J. J. (2002). Emotion regulation: affective, cognitive, and social consequences. Psychophysiology, 39(3), 281-291. doi: 10.1017.S0048577201393198

Gul, A., \& Khan, K. (2014). Emotion regulation strategies can predict task-switching abilities in euthymic bipolar patients. Front Hum Neurosci, 8, 847. doi:

10.3389/fnhum.2014.00847

Johnson, D. R. (2009). Emotional attention set-shifting and its relationship to anxiety and emotion regulation. Emotion, 9(5), 681-690. doi: 10.1037/a0017095

Koch, I., Gade, M., Schuch, S., \& Philipp, A. M. (2010). The role of inhibition in task switching: a review. Psychon Bull Rev, 17(1), 1-14. doi: 10.3758/PBR.17.1.1

Kret, M. E., \& Ploeger, A. (2015). Emotion processing deficits: a liability spectrum providing insight into comorbidity of mental disorders. Neurosci Biobehav Rev, 52, 153-171. doi: 10.1016/j.neubiorev.2015.02.011

Krug, M. K., \& Carter, C. S. (2012). Proactive and reactive control during emotional interference and its relationship to trait anxiety. Brain Res, 1481, 13-36. doi: $10.1016 /$ j.brainres.2012.08.045 
Leite, J., Carvalho, S., Fregni, F., Boggio, P. S., \& Goncalves, O. F. (2013). The effects of cross-hemispheric dorsolateral prefrontal cortex transcranial direct current stimulation (tDCS) on task switching. Brain Stimul, 6(4), 660-667. doi: 10.1016/j.brs.2012.10.006

Loose, R., Kaufmann, C., Tucha, O., Auer, D. P., \& Lange, K. W. (2006). Neural networks of response shifting: influence of task speed and stimulus material. Brain Res, 1090(1), 146-155. doi: 10.1016/j.brainres.2006.03.039

Malooly, A. M., Genet, J. J., \& Siemer, M. (2013). Individual differences in reappraisal effectiveness: the role of affective flexibility. Emotion, 13(2), 302-313. doi: $10.1037 / \mathrm{a} 0029980$

Meron, D., Hedger, N., Garner, M., \& Baldwin, D. S. (2015). Transcranial direct current stimulation (tDCS) in the treatment of depression: Systematic review and metaanalysis of efficacy and tolerability. Neurosci Biobehav Rev, 57, 46-62. doi: 10.1016/j.neubiorev.2015.07.012

Monsell, S., Sumner, P., \& Waters, H. (2003). Task-set reconfiguration with predictable and unpredictable task switches. Mem Cognit, 31(3), 327-342.

Monsell, S., Yeung, N., \& Azuma, R. (2000). Reconfiguration of task-set: is it easier to switch to the weaker task? Psychol Res, 63(3-4), 250-264.

Nitsche, M. A., \& Paulus, W. (2000). Excitability changes induced in the human motor cortex by weak transcranial direct current stimulation. J Physiol, 527 Pt 3, 633-639.

Piguet, C., Sterpenich, V., Desseilles, M., Cojan, Y., Bertschy, G., \& Vuilleumier, P. (2013). Neural substrates of cognitive switching and inhibition in a face processing task. Neuroimage, 82, 489-499. doi: 10.1016/j.neuroimage.2013.06.015

Ponizovsky, A. M., Finkelstein, I., Poliakova, I., Mostovoy, D., Goldberger, N., \& Rosca, P. (2013). Interpersonal distances, coping strategies and psychopathology in patients with depression and schizophrenia. World J Psychiatry, 3(3), 74-84. doi: 10.5498/wjp.v3.i3.74

Pripfl, J., \& Lamm, C. (2015). Focused transcranial direct current stimulation (tDCS) over the dorsolateral prefrontal cortex modulates specific domains of self-regulation. Neurosci Res, 91, 41-47. doi: 10.1016/j.neures.2014.09.007

Ravizza, S. M., \& Carter, C. S. (2008). Shifting set about task switching: behavioral and neural evidence for distinct forms of cognitive flexibility. Neuropsychologia, 46(12), 2924-2935. doi: 10.1016/j.neuropsychologia.2008.06.006

Reeck, C., \& Egner, T. (2014). Emotional task management: neural correlates of switching between affective and non-affective task-sets. Soc Cogn Affect Neurosci. doi: $10.1093 / \mathrm{scan} / \mathrm{nsu} 153$

Riva, P., Romero Lauro, L. J., DeWall, C. N., Chester, D. S., \& Bushman, B. J. (2015). Reducing aggressive responses to social exclusion using transcranial direct current stimulation. Soc Cogn Affect Neurosci, 10(3), 352-356. doi: 10.1093/scan/nsu053

Shiozawa, P., Fregni, F., Bensenor, I. M., Lotufo, P. A., Berlim, M. T., Daskalakis, J. Z., . . . Brunoni, A. R. (2014). Transcranial direct current stimulation for major depression: an updated systematic review and meta-analysis. Int J Neuropsychopharmacol, 17(9), 1443-1452. doi: 10.1017/S1461145714000418

Shobe, E. R. (2014). Independent and collaborative contributions of the cerebral hemispheres to emotional processing. Front Hum Neurosci, 8, 230. doi: 10.3389/fnhum.2014.00230

Spielberger, C. D., Reheiser, E. C., \& Sydeman, S. J. (1995). Measuring the experience, expression, and control of anger. Issues Compr Pediatr Nurs, 18(3), 207-232.

Tremblay, S., Lepage, J. F., Latulipe-Loiselle, A., Fregni, F., Pascual-Leone, A., \& Theoret, H. (2014). The uncertain outcome of prefrontal tDCS. Brain Stimul, 7(6), 773-783. doi: $10.1016 /$ j.brs.2014.10.003 
Vanderhasselt, M. A., De Raedt, R., Brunoni, A. R., Campanha, C., Baeken, C., Remue, J., \& Boggio, P. S. (2013). tDCS over the left prefrontal cortex enhances cognitive control for positive affective stimuli. PLoS One, 8(5), e62219. doi: 10.1371/journal.pone.0062219

Wendelken, C., Munakata, Y., Baym, C., Souza, M., \& Bunge, S. A. (2012). Flexible rule use: common neural substrates in children and adults. Dev Cogn Neurosci, 2(3), 329339. doi: 10.1016/j.den.2012.02.001

Willis, M. L., Murphy, J. M., Ridley, N. J., \& Vercammen, A. (2015). Anodal tDCS targeting the right orbitofrontal cortex enhances facial expression recognition. Soc Cogn Affect Neurosci. doi: $10.1093 / \mathrm{scan} / \mathrm{nsv} 057$

Wyman, P. A., Cross, W., Hendricks Brown, C., Yu, Q., Tu, X., \& Eberly, S. (2010). Intervention to strengthen emotional self-regulation in children with emerging mental health problems: proximal impact on school behavior. J Abnorm Child Psychol, 38(5), 707-720. doi: 10.1007/s10802-010-9398-x

Yoshida, W., Funakoshi, H., \& Ishii, S. (2010). Hierarchical rule switching in prefrontal cortex. Neuroimage, 50(1), 314-322. doi: 10.1016/j.neuroimage.2009.12.017 
Table 1. Mean reaction times during the affective switching task and total scores on self-reported questionnaires

\begin{tabular}{|c|c|c|c|c|c|c|}
\hline & \multicolumn{2}{|c|}{ Right tDCS } & \multicolumn{2}{|c|}{ Left tDCS } & \multicolumn{2}{|c|}{ Sham } \\
\hline & Emotion & Gender & Emotion & Gender & Emotion & Gender \\
\hline Task-Switch & gender-emotion & emotion-gender & gender-emotion & emotion-gender & gender-emotion & emotion-gender \\
\hline Block 1 & $746(107)$ & $670(78)$ & $740(118)$ & $718(103$ & $775(143)$ & 735 (136) \\
\hline Block 2 & $695(75)$ & 667 (69) & $686(134)$ & $699(142)$ & $734(142)$ & $719(152)$ \\
\hline Task-repetition & emotion-emotion & gender-gender & emotion-emotion & gender-gender & emotion-emotion & gender-gender \\
\hline Block 1 & $711(76)$ & $681(80)$ & $743(119)$ & $700(105)$ & 767 (132) & $742(157)$ \\
\hline Block 2 & $703(72)$ & $666(81)$ & $706(131)$ & $684(130)$ & $769(200)$ & $705(146)$ \\
\hline \multicolumn{7}{|l|}{ Questionnaires } \\
\hline Beck & \multicolumn{2}{|c|}{$8.0(8.59)$} & \multicolumn{2}{|c|}{$7.07(4.76)$} & \multicolumn{2}{|c|}{$5.93(5.82)$} \\
\hline$S T A X I-T$ & \multicolumn{2}{|c|}{$19.8(3.34)$} & \multicolumn{2}{|c|}{$17.7(3.64)$} & \multicolumn{2}{|c|}{$18.7(4.17)$} \\
\hline ERQ-Reappraisal & \multicolumn{2}{|c|}{$5.04(0.74)$} & \multicolumn{2}{|c|}{$4.72(0.97)$} & \multicolumn{2}{|c|}{$4.81(0.73)$} \\
\hline ERQ-Suppression & \multicolumn{2}{|c|}{$3.75(1.08)$} & \multicolumn{2}{|c|}{$3.64(1.28)$} & \multicolumn{2}{|c|}{$3.06(1.08)$} \\
\hline
\end{tabular}

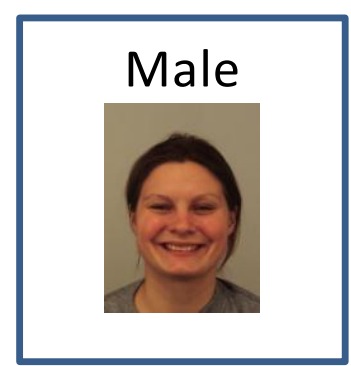

No-Go trial Gender task

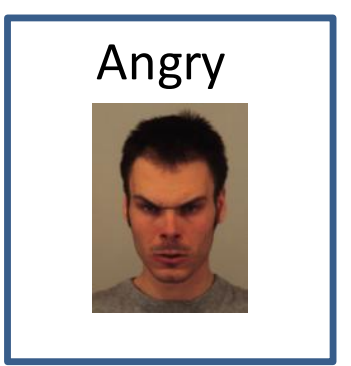

Go trial Emotion task

Figure 1. Example of stimuli 


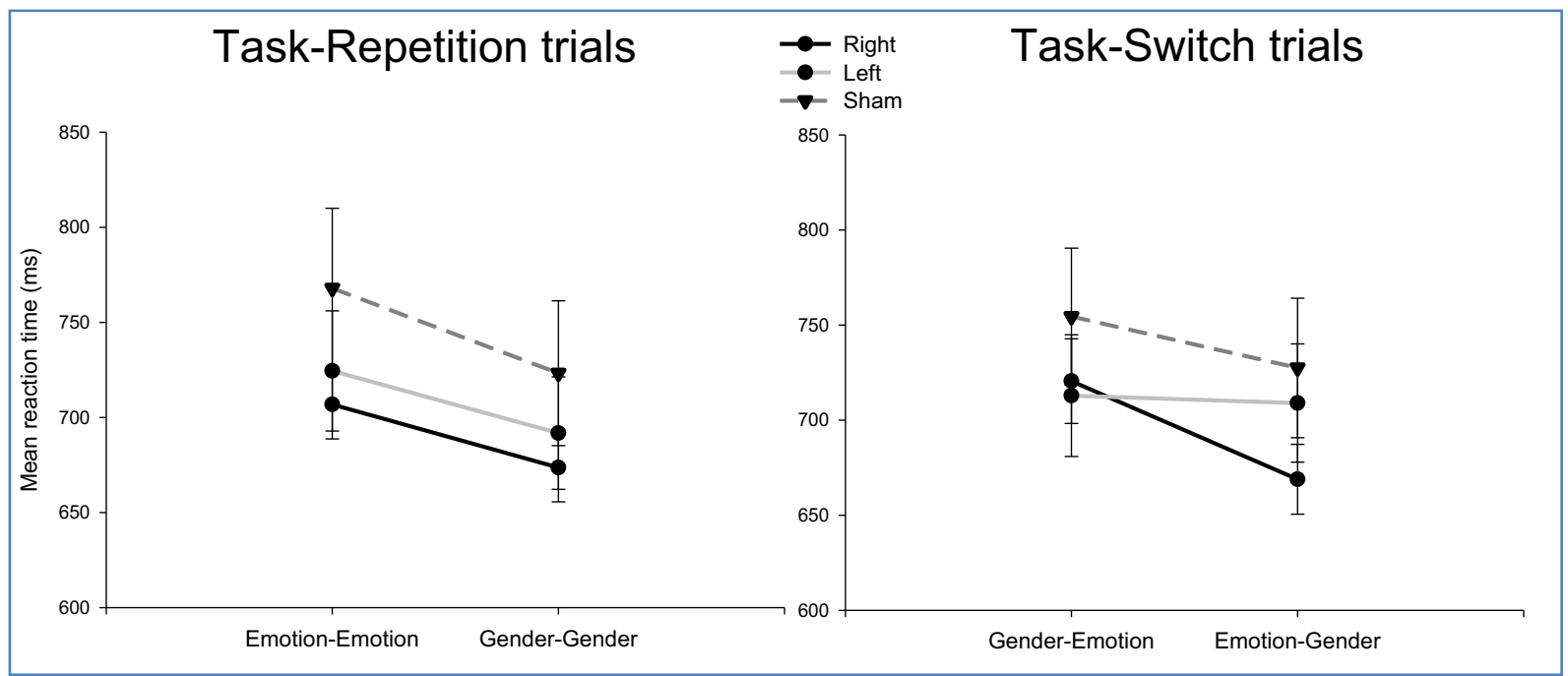

Figure 2. Mean reaction times for task-repetition and task-switch trials

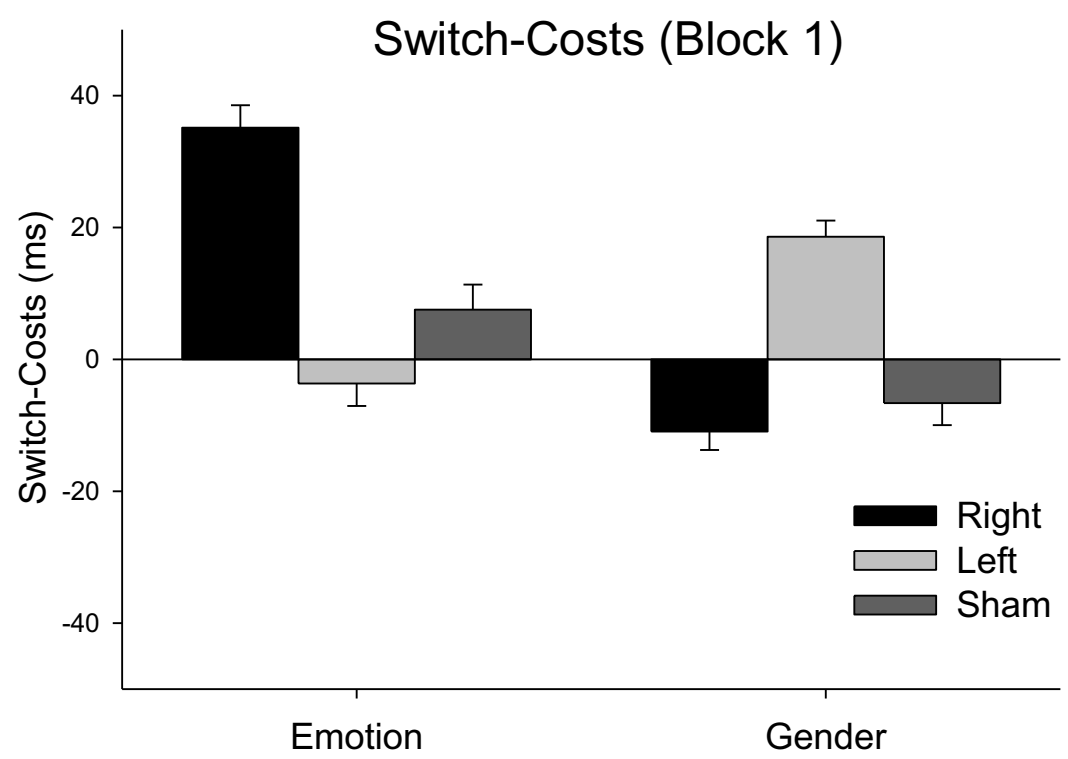

Figure 3. Switch-costs (difference between mean RT's for switch and repetition trials) for emotion and gender 